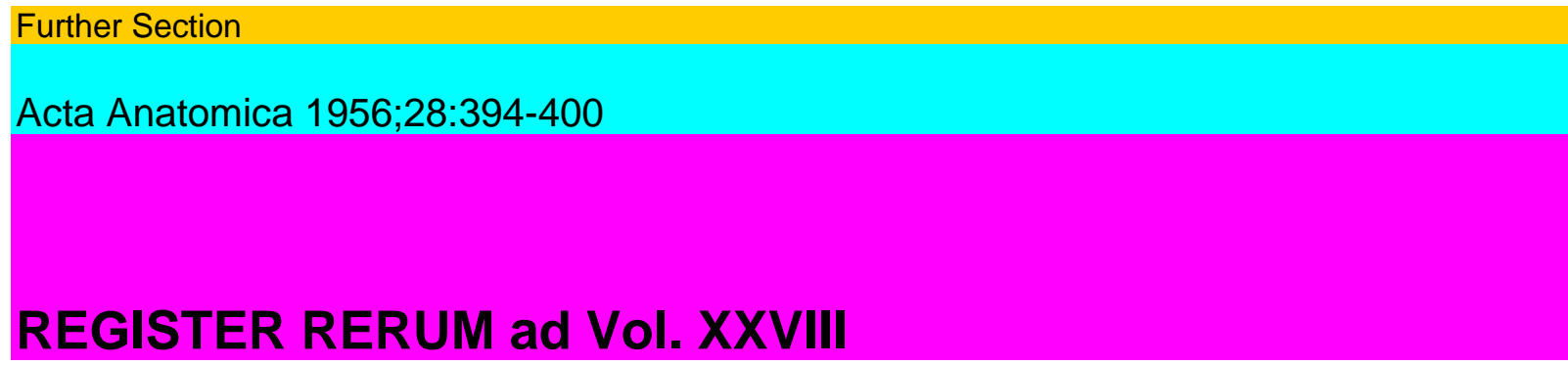

Confecit G. Boehm, Basel

Adrenals, v. Mitosenperiodik, Ossification

Adrenocortical interaction, v. Hexoestrol

Adventitia, v. Druckreiz-Rezeptoren

Ageing, v. Altern

Allocortex cerebri, v. Grauzellkoeffizient

Altern; qualitative und quantitative Untersuchung der Nervenzellen des

Ganglion coeliacum im Alter (Alterserscheinungen der sympathischen

Nervenzellen) 157

Ameloblasten, v. Dentes

Anal, nerf anal, caractéristiques (C) 387

Anatomen; Freie Vereinigung der Anatomen an Schweizerischen Hoch-

schulen, 22. Tagung (1956), Verhandlungen (C) $381 \mathrm{ff}$.

Anatomie individuelle, v. Thorax

Anencephalic human embryo of 33.5 millimetres $\quad 149$

Angle xyphoïdien, v. Thorax

Annulus fibrosus, v. Cor

Aorta; etude du rétablissement circulatoire après exclusion de la bifurcation aortique (C) 389

Aponeurosis of the prime movers of the right atrio-ventricular plane in the

human heart

93

Appareil digestif humain; observations comparatives sur Гinnervation de quelques regions de Tappareil digestif humain (C) 388

Arterien; Vorkommen und Entstehung von Längsmuskulatur in Arterien am

Beispiel der Bronchialarterien des Menschen (C) $\quad 390$

Atlas cerebri humani (B) 391

Atrio-ventricular plane, right, in the human heart, aponeurosis of its prime movers 93

Auris interna, v. Labyrinth

Autonomes Nervensystem, v. Ganglion coeliacum

Autoradiography, v. Dentes

Basal membrane, v. Skin of the newt

Batraciens, v. Neurosécrétion

Bauchmuskeln, v. Myotome

Bj-Avitaminose, v. Pankreas

Bifurcation aortique; contribution à $\Gamma$ étude du rétablissement circulatoire après exclusion de la bifurcation aortique (C) $\quad 389$

Bileducts, hepatic, v. Gallengänge

Biliaires, canaux biliaires intrahépatiques, v. Gallengänge 
Bindegewebsstrukturen, v. Testis

Biorhythmen, v. Mitosenperiodik

Bläschendrüse (Glandula vesiculosa) des Rindes, funktionelle Anatomie . . 359

Blutkreislauf, v. Aorta

1 (B) = Book Reviews - Livres nouveaux - Buchbesprechungen $(C)=$ Report or Demonstration

- Communication ou Demonstration -Vortrag oder Demonstration

Register rerum395

Bovís, v. Bläschendrüse

Brain; human brain cortex, v. Grauzellkoeffizient

Bronchialarterien des Menschen, v. Arterien

Bulbus olfactorius, v. Olfactorius

Bull, v. Bläschendrüse

Capillaries, v. Vascularity

Capsules of the joint, v. Druckreiz-Rezeptoren

Cavum nasi, v. Nasenhöhlen

391391

Celestino da Costa, A. $\dagger$

Cells, fine structure (B)

206391

Céphalopodes; nouvelles contributions à la connaissance du système nerveux

du tentacule des céphalopodes. III. Ganglion sous-acétabulaire et ses

rapports avec une pseudo-articulation de la ventouse

Cerebrum; Atlas cerebri humani (B)

Cerebrum, v. Grauzellkoeffizient, Neurosécrétion, Olfactorius

Chick embryo, development, v. Hyaluronidase

Choanen, v. Nasenhöhlen

Cholangiogramm, v. Gallengänge

Cholinesterase-Reaktion, v. Endplatten

Circulation du sang, v. Aorta

Cobaye, v. Ossification

Comptes rendus, v. Anatomen

Congrès, v. Anatomen

Coniocortex cerebri, v. Grauzellkoeffizient

93

Cor; aponeurosis of the prime movers of right atrio-ventricular plane in the

human heart

Cortex cerebri, v. Grauzellkoeffizient

Cortico-surrénale, v. Ossification

Crista supraventricularis, v. Cor

"Crooked-tail" bei der Maus, v. Wirbelsäule

Cultures de tissu, v. Muscles, Neurites

Cytoarchitektonik, v. Grauzellkoeffizient

Cytochemistry, v. Histochemistry

386

111217

Difference anatomique individuelle, analyse (C) 
Digestif, v. Appareil digestif humain

Dentes; development of enamel and dentine on the molars of the mouse, with an account of the enamel-free areas

$-\bullet$, historadiographic and autoradiographic researches on hard dental

tissues

Dentine formation, v. Dentes

Dorure, méthode de dorure, v. Druckreiz-Rezeptoren

Druckreiz-Rezeptoren; Rezeptoren der Eingeweide und Druckreiz-Rezeptoren der Gefäße im Lichte neuer Untersuchungen mit der Ruffinischen Goldchlorürmethode 100

Drüsengewebe, v. Bläschendrüse, Schläfendrüse Ductus hepaticus, v. Gallengänge Ductus subvesicalis, v. Gallengänge Duodenalepithel der Ratte, v. Mitosenperiodik 396

Register rerum

Ecorce cérébrale, v. Grauzellkoeffizient

Eingeweide; die Druckreiz-Rezeptoren der Eingeweide und Gefäße im Licht

neuerer Untersuchungen mit der Ruffinischen Goldchlorürmethode . 100 Elefant, afrikanischer

und indischer, v. Schläfendrüse Elephas maximus, v. Schläfendrüse Email des dents,

différenciation histologiques, v. Dentes

Embryo, human, anencephalic, of 33.5 millimetres 149

- , v. Nasenhöhlen

Enamel organ, development, v. Dentes

Endplatten, motorische, ihre Lage innerhalb der Skeletmuskeln und die

multiple Innervation der einzelnen Muskelfasern (C) 384

Epithel, v. Mitosenperiodik

Extremitates, v. Metatarsus, Muscles jambier antérieur

Fasciculi bulbi dextri, v. Cor

Feinbau, v. Fine structure

Fel, v. Gallengänge

Fente de Larrey, v. Thorax

Ferments, v. Endplatten, Hyaluronidase, Olfactorius

Fine structure of cells (B) 391

Foie; canaux biliaires, v. Gallengänge Funktionelle Anatomie, v. Bläschendrüse

Gallengänge des Menschen, systematische Anatomie (Röntgenanatomische

Studie)

Gallus domesticus, v. Hyaluronidase

Ganglion coeliacum; qualitative und quantitative Untersuchung der Ner-

venzellen des Ganglion coeliacum im Alter (Alterserscheinungen der sym-

pathischen Nervenzellen) 157

Ganglion subacetabulare, v. Céphalopodes

Gaumen, v. Nasenhöhlen

Gefäße; Druckreiz-Rezeptoren der Gefäße und der Eingeweide im Lichte

neuerer Untersuchungen mittels der Ruffinischen Goldchlorürmethode 100

Gefäße und Implantation (C) 388

Gefäße, v. Aorta, Arterien

Gefäßversorgung, v. Vascularity 
Gelenkkapsel, v. Druckreiz-Rezeptoren

Gerontologie, v. Altern

Geschlechtsdrüse, accessorische, des Rindes, v. Bläschendrüse

Gilding, method of gilding, v. Druckreiz-Rezeptoren

Glande temporale des elephants des Indes et d'Afrique, v. Schläfendrüse

Glandula vesiculosa (Bläschendrüse) des Rindes, funktionelle Anatomie . . 359

Goldchlorürmethode (Ruffiní), v. Druckreiz-Rezeptoren

Goldhamster, v. Implantation

Golgi-Mazzonísche Körperchen, v. Druckreiz-Rezeptoren

Grauzellkoeffizient der menschlichen Hirnrinde. Berechnungen nach dem

Zahlenmaterial v. Economo, s (II. Weitere Zusammenhänge und Berech-

nung der Gesamtzellzahl der Hirnrinde) 259

Greycell coefficient, v. Grauzellkoeffizient

Register rerum

397

Griseum, v. Grauzellkoeffizient Guinea-pig, v. Ossification

Haut von Triturus víridescens, v. Reticular network

Heart; aponeurosis of the prime movers of right atrio-ventricular plane in

the human heart

93

Hepatic bileducts, v. Gallengänge

Heredopathie, v. Wirbelsäule

Herzskelet, v. Cor

Hexoestrol; effect of hexoestrol on the thymus and the importance of adrenocortical interaction (Reply to H. Selye and D. Marion) 155

Histioradiography, v. Dentes

Histochemistry, v. Endplatten, Olfactorius

Hoden, v. Testis

Hormone, v. Thymus

Hühnerembryonen, Entwicklung, v. Hyaluronidase

Hyaluronidase, action sur le développement embryonnaire du poulet . . . 287

Hypophysis, v. Neurosécrétion

Hypothalamus, v. Neurosécrétion

Implantation und Gefäße (C) 388

Innervation, v. Appareil digestif humain

Innervation, multiple, der einzelnen Muskelfasern, v. Endplatten

Isocortex cerebri, v. Grauzellkoeffizient

Isotope, radioaktive, v. Dentes

Kaninchen, v. Vascularity Kapillarisation, v. Vascularity

Labyrinth; das eigenartige Gewebe des membranösen Labyrinths (C) . . . 385

Langerhanssche Inseln, v. Pankreas

Lapin, v. Vascularity

Larrey, fente de Larrßy, v. Thorax

Leber, v. Gallengänge

Liver; hepatic bileducts, v. Gallengänge

Loxodonta africana, v. Schläfendrüse

Mammalia, v. Olfactorius 
Man, v. Anencephalic embryo, Appareil digestif, Arterien, Atlas cerebri humani, Gallengänge, Grauzellkoeffizient, Heart, Myotome,Nasenhöhlen, «Sarkoplasmatisches Reticulum», Testis, Thorax Maus; formale Genese einer vererbten Wirbelsäulenmißbildung - die Mutante "crooked-tail" der Maus (C) 390

Mazzonísche Körperchen, v. Druckreiz-Rezeptoren

Meerschweinchen, v. Ossification

Membrana basilaris, v. Skin of the newt

Mesocricetus auratus, v. Implantation

Metatarsus; note sur Гasymétrie des têtes métatarsiennes (à Гexception de la premiere) (C) 390

398

\section{Register rerum}

Methode, v. Mikrotom

Mikrotom; technische Verbesserungen am Schlittenmikrotom (C) 382

Minerals, distribution, v. Dentes

Mißbildung, v. Anencephalic embryo, Hyaluronidase, Wirbelsäule

Mitosenperiodik in der Nebennierenrinde, Schilddrüse, im Duodenal- und

Mundhöhlenepithel der Ratte 331

Molars of the mouse, v. Dentes

Mundhöhlenepitbel der Ratte, v. Mitosenperiodik

Muscles; croissance de bourgeons de fibres musculaires en train de régénérer in vitro (film) (C) 390

-, jambier antérieur et long péronier lateral, analogie morphologique et antagonisme fonctionnel (C) 387

-, vascularity of some red and white muscles of the rabbit 342

-, v. Endplatten, Muskelfasern, Myotome

Musculi pectinati, v. Cor

Musculus peronaeus (= fibularis) longus, v. Muscles jambiers antérieur

Muskelfasern, quergestreifte; «sarkoplasmatisches Reticulum» in den quergestreiften Muskelfasern der Wirbeltiere und des Menschen 135

Muskeln, v. Muscles

Muskulatur (Längsmuskulatur), v. Arterien

Mus musculus, v. Dentes, Maus

Myotome; Differenzierung der Rumpfmyotome beim Menschen und die Herkunft der Bauchmuskeln (C) 383

Nasenhöhlen und Gaumen, Demonstration zu ihrer Entwicklung an mensch-

lichen Embryonen von 13-37 mm SSL (C) 390

Nebennierenrinde, v. Mitosenperiodik

Necrologia 391

Nerf anal, caractéristiques (C) 387

Nerven (Nervensystem, Zentralnervensystem), v. Appareil digestif, Cépha-

lopodes, Cerebrum, Endplatten, Grauzellkoeffizient, Nerf anal, Nerven-

zellen, Neurites, Neurosécrétion Nervenzellen des Ganglion coeliacum im Alter, qualitative und quantitative

Untersuchung (Alterserscheínungen der sympathischen Nervenzellen) . 157 
Netzhautpräparate, neue Befunde (C)

Neurites; mouvement amiboïde et pinocytose dans les neurites qui poussent dans les cultures (film) (C) 390

Neurosécrétion; les voies extra-hypophysaires des noyaux neurosécrétoires hypothalamiques chez les Batraciens et les Reptiles (C) 384

Nidation, v. Implantation

Octopus vulgaris, v. Céphalopodes

Odontoblasten, v. Dentes

Olfactorius; histochemische Succinodehydrogenase-Reaktíon im olfaktorischen System bei verschiedenen Säugetiergehirnen (C) 385

Ontogenesis, v. Anencephalic embryo, Dentes, Hyaluronidase, Myotome, Nasenhöhlen

Ossification dans la cortico-surrénale d'un cobaye, un cas (C)

Register rerum

399

Pacinische Körperchen, v. Druckreiz-Rezeptoren

Palatum, v. Nasenhöhlen

Pankreas der Ratte, Einfluß der BI-Avitaminose auf den Inselapparat und auf das exokrine Drüsengewebe (C) 382

Peau du triton, v. Reticular network

Pes, v. Metatarsus

Pied, v. Metatarsus

Pinocytose, v. Neurites

Poulet, embryon, développement, v. Hyaluronidase

Pression; sensation de pression, récepteurs, v. Druckreiz-Rezeptoren

Pseudo-articulation de la ventouse (Octopus), v. Céphalopodes

Pseudo-joint cavity (suctorial disc of Octopus), v. Céphalopodes

«Querfadennetz» (Muskeln), v. «Sarkoplasmatisches Reticulum»

Rabbit, v. Vascularity

Rat, v. Mitosenperiodik, Pankreas

Receptors (pressure), v. Druckreiz-Rezeptoren

Reptiles, v. Neurosécrétion

Reticular network, adepidermal, in the skin of the newt, Tríturus viridescens 313

Reticulum, v. «Sarkoplasmatisches Reticulum»

Retina, v. Netzhautpräparate

Rezeptoren, v. Druckreiz-Rezeptoren

Rhythmusforschung, v. Mitosenperiodik

Rind, v. Bläschendrüse

Röntgenanatomische Studie, v. Gallengänge

Ruffinische Körperchen, v. Druckreiz-Rezeptoren

Rumpfmyotome, v. Myotome

«Sarkoplasmatisches Reticulum» in den quergestreiften Muskelfasern der

Wirbeltiere und des Menschen $\quad 135$

Saugnapf, v. Céphalopodes

Schilddrüse, v. Mitosenperiodik

Schläfendrüse beim afrikanischen und indischen Elefanten (Loxodonta 
africana Cuvier und Elephas maximus L.), Feinbau 303

Segmenta hepatis, v. Gallengänge

Sexual gland, accessory, of the bull, v. Glandula vesiculosa

Skin of the newt; adepidermal reticular network in the skin of the newt,

Tríturus viridescens 313

Société, v. Anatomen

Statistische Auswertung, v. Mitosenperiodik

Stilboestrol, v. Hexoestrol

Submicroscopical structure, v. Fine structure

Substantia adamantina, v. Dentes

Succinodehydrogenase-Reaktion, v. Olfactorius

Suctorial disc, v. Céphalopodes

Sympathische Nervenzellen, v. Ganglion coeliacum

Systema digestivum, v. Appareil digestif

400

Register rerum

Taureau, v. Bläschendrüse

Teeth, v. Dentes

Temporal glands of indian and african elefants, v. Schläfendrüse

Tentacule des céphalopodes, v. Céphalopodes

Teratologie, v. Anencephalic embryo, Hyaluronidase, Wirbelsäule

Testis; Bindegewebsstrukturen am Mediastinalkörper des menschlichen

Hodens (C) 383

Thorax; analyse d'une difference anatomique individuelle (C) 386

Thymus; effect of hexoestrol on the thymus and the importance of adreno-

cortical interaction (Reply to H. Selye and D. Marion) 155

Thyreoidea, v. Mitosenperiodik Tissue cultures, v. Muscles, Neurites Tractus olfactoris, v.

Olfactorius Triturus vírídescens, v. Reticular network

Vaisseaux, v. Arterien, Aorta, Gefäße, Vascularity

Vascularity of some red and white muscles of the rabbit

Vater- Pacinísche Körperchen, v. Druckreiz-Rezeptoren

Vegetatives Nervensystem, v. Ganglion coeliacum

«Ventilebene» (atrio-ventricular plane), v. Heart

Ventouses, v. Céphalopodes

Ventriculus dexter, v. Cor

Verdauungstrakt, v. Appareil digestif humain

Verhandlungsbericht, v. Anatomen

Vertebra, v. Wirbelsäule

Vertebrata, v. «Sarkoplasmatisches Reticulum»

Vesicula seminalis, v. Glandula vesiculosa

Vesicular gland of the bull, v. Glandula vesiculosa

Vessels, v. Aorta, Arterien, Gefäße, Vascularity

Viscera, v. Druckreiz-Rezeptoren

Viszerale Rezeptoren, v. Druckreiz-Rezeptoren

Vitamin Bx, v. Pankreas

Wirbelsäule; formale Genese einer vererbten Wirbelsäulenmißbildung - die 
Mutante «crooked-tail» der Maus (C) 390

X-ray absorption (histioradiography), v. Dentes

$\mathrm{X}$-rays, v. Gallengänge

Xyphoïdien, angle xyphoïdien, v. Thorax

Zellen, v. Cells

Zellteilung, v. Mitosenperiodik

Zyto ..., v. Cyto ...

News from Universities - Nouvelles universitaires - Universitätsnachrichten

Eränkö, 0

393 Patzelt, V

393

Harrison, R. G

393 Pesonen, N

393

Jansen, J

393 Schenk, R

393

Kohn, A

393 